\title{
On a Class of Variational-Hemivariational Inequalities Involving Upper Semicontinuous Set-Valued Mappings
}

\author{
Guo-ji Tang, ${ }^{1}$ Zhong-bao Wang, ${ }^{2}$ and Hong-ling Zhang ${ }^{2}$ \\ ${ }^{1}$ School of Science, Guangxi University for Nationalities, Nanning, Guangxi 530006, China \\ ${ }^{2}$ Department of Mathematics, Southwest Jiaotong University, Chengdu 611756, China
}

Correspondence should be addressed to Zhong-bao Wang; zhongbaowang@hotmail.com

Received 24 January 2014; Accepted 17 March 2014; Published 27 April 2014

Academic Editor: Fu-quan Xia

Copyright (c) 2014 Guo-ji Tang et al. This is an open access article distributed under the Creative Commons Attribution License, which permits unrestricted use, distribution, and reproduction in any medium, provided the original work is properly cited.

\begin{abstract}
This paper is devoted to the various coercivity conditions in order to guarantee existence of solutions and boundedness of the solution set for the variational-hemivariational inequalities involving upper semicontinuous operators. The results presented in this paper generalize and improve some known results.
\end{abstract}

\section{Introduction}

Let $K$ be a nonempty, closed, and convex subset in $\mathbb{R}^{n}$. Let $F: K \rightrightarrows \mathbb{R}^{n}$ be a set-valued mapping and let $\phi: \mathbb{R}^{n} \rightarrow$ $\mathbb{R} \cup\{+\infty\}$ be a convex and lower semicontinuous function such that $K_{\phi}:=K \cap \operatorname{dom} \phi \neq \emptyset$, where $\operatorname{dom} \phi:=\left\{x \in \mathbb{R}^{n}\right.$ : $\phi(x)<+\infty\}$ is the effective domain of $\phi$. Let $\Omega$ be a bounded open set in $\mathbb{R}^{N}$ and $j(x, y): \Omega \times \mathbb{R}^{k} \rightarrow \mathbb{R}$ be a function. Let $T: \mathbb{R}^{n} \rightarrow L^{p}\left(\Omega ; \mathbb{R}^{k}\right)$ be a linear and continuous mapping, where $1<p<\infty$. We shall denote $\widehat{u}:=T u$ and denote by $j^{\circ}(x, y ; h)$ Clarke's generalized directional derivative of a locally Lipschitz mapping $j(x, \cdot)$ at the point $y \in \mathbb{R}^{k}$ with respect to the direction $h \in \mathbb{R}^{k}$, where $x \in \Omega$. In this paper, we consider the following variational-hemivariational inequality problems:

(P) find $u \in K_{\phi}$ and $u^{*} \in F(u)$ such that

$$
\begin{aligned}
& \left\langle u^{*}, v-u\right\rangle+\phi(v)-\phi(u) \\
& \quad+\int_{\Omega} j^{\circ}(x, \widehat{u}(x) ; \widehat{v}(x)-\widehat{u}(x)) d x \geq 0, \quad \forall v \in K,
\end{aligned}
$$

which is studied by some researchers (see, for example, $[1,2]$ ). Problem (P) includes some models as special cases.
Case 1. In the case when $F$ is single-valued, problem (P) becomes the following variational-hemivariational inequality problem: find $u \in K$ such that

$$
\begin{aligned}
& \langle F(u), v-u\rangle+\phi(v)-\phi(u) \\
& \quad+\int_{\Omega} j^{\circ}(x, \widehat{u}(x) ; \widehat{v}(x)-\widehat{u}(x)) d x \geq 0, \quad \forall v \in K .
\end{aligned}
$$

In 2000, by using Mosco's Theorem, Motreanu and Rădulescu [3] proved that if the operator $F$ is monotone and hemicontinuous, then problem (2) admits a solution (see Theorem 2 of [3]).

Case 2. If $\phi=I_{K}$, where $I_{K}$ is the indicator function over the set $K$, that is, $I_{K}(x)=0$ if $x \in K$ and $I_{K}(x)=+\infty$ otherwise, then problem $(\mathrm{P})$ reduces to the following hemivariational inequality: find $u \in K$ and $u^{*} \in F(u)$ such that

$$
\left\langle u^{*}, v-u\right\rangle+\int_{\Omega} j^{\circ}(x, \widehat{u}(x) ; \widehat{v}(x)-\widehat{u}(x)) d x \geq 0, \quad \forall v \in K,
$$

which is studied recently by Zhang and $\mathrm{He}[4,5]$. In 2011, by introducing the notion of stable quasimonotonicity and applying KKM theorem, Zhang and He [4] obtained some existence results of the hemivariational inequality (3). 
Case 3. If $F$ is single-valued and $\phi=I_{K}$, then problem (P) reduces to the following hemivariational inequality of finding $u \in K$ such that

$$
\langle F(u), v-u\rangle+\int_{\Omega} j^{\circ}(x, \widehat{u}(x) ; \widehat{v}(x)-\widehat{u}(x)) d x \geq 0,
$$

$\forall v \in K$,

which is introduced and named as Hartman-Stampacchia type hemivariational inequality by Panagiotopoulos et al. [6] and further studied by Costea and Rădulescu [7]. Under some suitable assumptions, the authors obtained corresponding existence theorems.

Case 4. If $T \equiv 0$, then problem (P) is equivalent to finding $u \in K$ and $u^{*} \in F(u)$ such that

$$
\left\langle u^{*}, v-u\right\rangle+\phi(v)-\phi(u) \geq 0, \quad \forall v \in K,
$$

which is called the generalized mixed variational inequality problem and intensively studied by many researchers (see, e.g., [8-12]). Further, if $F$ is single-valued and $\phi=I_{K}$, then problem (5) reduces to well-known formulation of variational inequality: find $u \in K$ such that

$$
\langle F(u), v-u\rangle \geq 0, \quad \forall v \in K .
$$

The notion of the hemivariational inequality was introduced by Panagiotopoulos (see, e.g., [13-16]) in the early 1980 s as variational expressions for several classes of mechanical problems with nonsmooth and nonconvex energy superpotentials. The derivative of hemivariational inequality is based on the mathematical notion of the generalized gradient of Clarke (see [17]). The hemivariational inequalities appear in a variety of mechanical problems, for example, the unilateral contact problems in nonlinear elasticity, the problems describing the adhesive and frictional effects, the nonconvex semipermeability problems, the masonry structures, and the delamination problems in multilayered composites; see $[14,16,18]$ for detailed descriptions. Extensive attention has been paid to the existence results for some types of hemivariational inequalities by many researchers in recent years. For example, Carl [19], Carl et al. [20, 21], and Xiao and Huang [22] studied the existence of solutions of some kinds of hemivariational inequalities using the method of sub-super solutions. Migórski and Ochal [23] and Park and Ha [24, 25] studied the problem using the regularized approximating method. Goeleven et al. [26] and Liu [27] proved the existence of solutions using the method of the first eigenfunction. For more related works regarding the existence of solutions for hemivariational inequalities, we refer to $[1,3,6,14-16,28-30]$ and the references therein.

Due to the presence of a set-valued mapping, problem (P) becomes more difficult than the single-valued case. First, we recall some definitions of continuity for set-valued mapping.

Definition 1 . The set-valued mapping $F: K \rightrightarrows \mathbb{R}^{n}$ is said to be the following:

(i) lower semicontinuous at $x_{0}$ if, for any $x_{0}^{*} \in F\left(x_{0}\right)$ and sequence $\left\{x_{n}\right\} \subset K$ with $x_{n} \rightarrow x_{0}$, a sequence $x_{n}^{*} \in F\left(x_{n}\right)$ can be determined which converges to $x_{0}^{*}$. If this is true at every $x_{0} \in K$, we say that $F$ is lower semicontinuous on $K$;

(ii) lower hemicontinuous if the restriction of $F$ to every line segment of $K$ is lower semicontinuous;

(iii) upper semicontinuous if, for all $x \in K$ and for any open set $W \subset \mathbb{R}^{n}$ satisfying $F(x) \subset W$, there exists an open neighborhood $U$ of $x$ such that $F(y) \subset W$ for all $y \in U \cap K$.

We remark that when $F$ is single-valued, both the notion of lower semicontinuity and that of upper semicontinuity coincide with the usual notion of continuity of a map.

When the constrained set $K$ is unbounded, in order to obtain existence theorems of problems, various of coercivity conditions usually are required (see, e.g., [2, 4, 5, 31-34]).

Recently, Tang and Huang [2] introduced some coercivity conditions for problem (P) involving lower hemicontinuous mappings. Using $\phi$-quasimonotonicity of mappings, the authors obtained some existence theorems and studied the boundedness of the solution set of problem (P). A natural problem is whether these coercivity conditions are valid for problem (P) involving upper semicontinuous mappings or not. This is the main motivation of this paper. On the other hand, Zhang and He [5] also investigated problem (3) involving upper semicontinuous mappings. How to extend the main results of [5] from problem (3) to problem (P) is another motivation of this work.

Motivated and inspired by the research work mentioned above, in this paper, we investigate various coercivity conditions in order to guarantee existence of solutions and boundedness of the solution set for the variational-hemivariational inequalities involving upper semicontinuous operators. The results presented in this paper generalize and improve some known results.

\section{Preliminaries}

For a nonempty, closed, and convex subset $K$ of a Euclidean space $\mathbb{R}^{n}$ and every $r>0$, we define

$$
B_{r}:=\{u \in K:\|u\| \leq r\} .
$$

Let $T: \mathbb{R}^{n} \rightarrow L^{p}\left(\Omega ; \mathbb{R}^{k}\right)$ be a linear compact operator, where $1<p<\infty$ and $k \geq 1$, and let $\Omega$ be a bounded open set in $\mathbb{R}^{N}$. Denote by $q$ the conjugated exponent of $p$; that is, $(1 / p)+$ $(1 / q)=1$. Let $j: \Omega \times \mathbb{R}^{k} \rightarrow \mathbb{R}$ be a function such that the mapping

$$
j(\cdot, y): \Omega \longrightarrow \mathbb{R} \text { is measurable, for every } y \in \mathbb{R}^{k} .
$$

We assume that at least one of the following conditions holds: either there exists $l \in L^{q}(\Omega ; \mathbb{R})$ such that

$$
\begin{array}{r}
\left|j\left(x, y_{1}\right)-j\left(x, y_{2}\right)\right| \leq l(x)\left|y_{1}-y_{2}\right|, \\
\forall x \in \Omega, \quad \forall y_{1}, y_{2} \in \mathbb{R}^{k},
\end{array}
$$

or

the mapping $j(x, \cdot)$ is locally Lipschitz, $\quad \forall x \in \Omega$, 
and there exists $C>0$ such that

$$
|z| \leq C\left(1+|y|^{p-1}\right), \quad \forall x \in \Omega, \forall z \in \partial j(x, y) .
$$

Recall that $f^{\circ}(x ; v)$ denotes Clarke's generalized directional derivative of the locally Lipschitz mapping $f: \mathbb{R}^{n} \rightarrow$ $\mathbb{R}$ at the point $x \in \mathbb{R}^{n}$ with respect to the direction $v \in \mathbb{R}^{n}$, while $\partial f(x)$ is the Clarke's generalized gradient of $f$ at $x \in \mathbb{R}^{n}$ (see, e.g., [17]); that is,

$$
\begin{gathered}
f^{\circ}(x ; v)=\limsup _{y \rightarrow x, t \rightarrow 0^{+}} \frac{f(y+t v)-f(y)}{t}, \\
\partial f(x)=\left\{\xi \in \mathbb{R}^{n}:\langle\xi, v\rangle \leq f^{\circ}(x ; v), \forall v \in \mathbb{R}^{n}\right\} .
\end{gathered}
$$

Let $J: L^{p}\left(\Omega ; \mathbb{R}^{k}\right) \rightarrow \mathbb{R}$ be an arbitrary locally Lipschitz functional. For each $u \in \mathbb{R}^{n}$ there exists (see, e.g., [17]) $z_{u} \in$ $\partial J(\widehat{u})$ such that

$$
J^{\circ}(\widehat{u} ; \xi)=\left\langle z_{u}, \xi\right\rangle=\max \{\langle w, \xi\rangle: \xi \in \partial J(\widehat{u})\} .
$$

Lemma 2 (Proposition 2.1.1 of [17]). Let $f: K \rightarrow \mathbb{R}$ be Lipschitz of rank $M$ near $x$. Then

(i) the function $v \rightarrow f^{\circ}(x ; v)$ is finite, positively homogeneous, and subadditive on $\mathbb{R}^{n}$ and satisfies

$$
\left|f^{\circ}(x ; v)\right| \leq M\|v\|
$$

(ii) $f^{\circ}(x ; v)$ is upper semicontinuous as a function of $(x, v)$ and, as a function of $v$ alone, is Lipschitz of rank $M$ on $\mathbb{R}^{n}$;

(iii) $f^{\circ}(x ;-v)=(-f)^{\circ}(x ; v)$.

Lemma 3 (Propositions 2.1.2 and 2.1.5 of [17]). Let $f: K \rightarrow$ $\mathbb{R}$ be Lipschitz of rank $M$ near $x$. Then

(i) $\partial f(x)$ is a nonempty, convex, and weak ${ }^{*}$-compact subset of $X^{*}$ and $\|\zeta\|_{*} \leq M$ for every $\zeta$ in $\partial f(x)$;

(ii) for every $v$ in $\mathbb{R}^{n}$, one has

$$
f^{\circ}(x ; v)=\max \{\langle\zeta, v\rangle: \zeta \in \partial f(x)\}
$$

(iii) $\partial f$ is upper semicontinuous at $x$.

Lemma 4 (Theorem 2.7.5 of [17]). If $J(\varphi)=\int_{\Omega} j(x, \varphi(x)) d x$ and $j$ satisfies the conditions (8) and (9) or (8) and (10)-(11), then $J$ is uniformly Lipschitz on bounded subsets, and one has

$$
\partial J(\varphi) \subset \int_{\Omega} \partial j(x, \varphi(x)) d x .
$$

Further, if $j$ is regular at $(x, \varphi(x))$ then $J$ is regular at $\varphi$ and equality holds.

Lemma 5 (Proposition 24.1 of [35]). Let X, Y be Banach spaces and let $F$ be an upper semicontinuous set-valued mapping with nonempty compact values from $X$ to $Y$. Then for any compact subset $E$ of $X, F(E)$ is compact.
Lemma 6 (Theorem 4.1 of [10]). Assume that $\mathbb{B}$ is a reflexive, strictly convex, and smooth Banach space with the dual space $\mathbb{B}^{*}$ and $\mathbb{B}$ has property $(h): x_{n} \rightarrow x$ weakly and $\left\|x_{n}\right\| \rightarrow\|x\|$ imply that $x_{n} \rightarrow x$. Suppose that $K \subset \mathbb{B}$ is nonempty closed convex set and $\phi: \mathbb{B} \rightarrow \mathbb{R} \cup\{+\infty\}$ is proper, convex, and lower semicontinuous. Let $F(x)=J(x)-T(x)$ for all $x \in K$, where $J: \mathbb{B} \rightrightarrows \mathbb{B}^{*}$ is normalized duality mapping and $T: K \rightrightarrows \mathbb{B}^{*}$ is a compact mapping with compact convex values. Suppose that there exists a vector $\hat{x} \in K$ such that $\phi(\hat{x})=\inf _{x \in K_{\phi}} \phi(x)$ and the set

$$
L_{<}^{\phi}(\widehat{x}):=\left\{x \in K_{\phi}: \inf _{x^{*} \in F(x)}\left\langle x^{*}, x-\widehat{x}\right\rangle+\phi(x)-\phi(\widehat{x})<0\right\}
$$

is bounded (possibly empty). Then there exists $x_{0} \in K_{\phi}$ and $x_{0}^{*} \in F\left(x_{0}\right)$ such that

$$
\left\langle x_{0}^{*}, y-x_{0}\right\rangle+\phi(y)-\phi\left(x_{0}\right) \geq 0, \quad \forall y \in K .
$$

Theorem 7. Let $K$ be a closed and convex subset of $\mathbb{R}^{n}$. Let $F$ : $K \rightrightarrows \mathbb{R}^{n}$ be an upper semicontinuous set-valued mapping with compact convex values and let $\phi: \mathbb{R}^{n} \rightarrow \mathbb{R} \cup\{+\infty\}$ be a convex and lower semicontinuous function such that $K_{\phi} \neq \emptyset$. Suppose that there exists a vector $\widehat{x} \in K_{\phi}$ such that $\phi(\widehat{x})=\inf _{x \in K_{\phi}} \phi(x)$ and the set

$$
L_{<}^{\phi}(\widehat{x}):=\left\{x \in K_{\phi}: \inf _{x^{*} \in F(x)}\left\langle x^{*}, x-\widehat{x}\right\rangle+\phi(x)-\phi(\widehat{x})<0\right\}
$$

is bounded (possibly empty). Then there exists $x_{0} \in K_{\phi}$ and $x_{0}^{*} \in F\left(x_{0}\right)$ such that

$$
\left\langle x_{0}^{*}, y-x_{0}\right\rangle+\phi(y)-\phi\left(x_{0}\right) \geq 0, \quad \forall y \in K .
$$

Proof. For any bounded set $A \subset K$, we have $\overline{F(A)} \subset \overline{F(\bar{A})}$. Since $F: K \rightrightarrows \mathbb{R}^{n}$ is an upper semicontinuous mapping with compact convex values, by Lemma 5 , we obtain that $\overline{F(\bar{A})}$ is a compact set and so $\overline{F(A)}$ is compact. Hence $F$ is a compact mapping with compact convex values. Taking $T(x)=x-$ $F(x)$ for any $x \in K$ and applying Lemma 6 , we obtain the conclusion.

Remark 8. Theorem 7 generalizes Theorem 3.1 of Qiao and $\mathrm{He}$ [36] (see also Corollary 4.2 of [10]) from set-valued variational inequalities to set-valued mixed variational inequalities. Moreover, it also generalizes the corresponding result of Facchinei and Pang [33] (see Proposition 2.2.3 of [33]). This theorem plays a crucial role in analysis of the next section.

\section{Coercivity Conditions and Applications to Existence Theorems}

First, we consider another type of variational-hemivariational inequality problem:

$\left(\mathrm{P}^{\prime}\right)$ find $u \in K_{\phi}$ and $u^{*} \in F(u)$ such that

$$
\left\langle u^{*}, v-u\right\rangle+\phi(v)-\phi(u)+J^{\circ}(\widehat{u} ; \widehat{v}-\widehat{u}) \geq 0, \quad \forall v \in K,
$$

where $J: L^{p}\left(\Omega ; \mathbb{R}^{k}\right) \rightarrow \mathbb{R}$ is a locally Lipschitz functional. 
We denote by $S$ (resp. $S^{\prime}$ ) the solution set of problem (P) (resp., $\left.\left(\mathrm{P}^{\prime}\right)\right)$.

Proposition 9. Let $J: L^{p}\left(\Omega ; \mathbb{R}^{k}\right) \rightarrow \mathbb{R}$ be the function

$$
J(\varphi)=\int_{\Omega} j(x, \varphi(x)) d x .
$$

And let $T: \mathbb{R}^{n} \rightarrow L^{p}\left(\Omega ; \mathbb{R}^{k}\right)$ be a linear compact operator, where $1<p<\infty, k \geq 1$, and $\Omega$ is a bounded open set in $\mathbb{R}^{N}$. Assume that $K$ is a nonempty, closed, and convex subset of $\mathbb{R}^{n}$. Let $F: K \rightrightarrows \mathbb{R}^{n}$ be a set-valued mapping and let $\phi: \mathbb{R}^{n} \rightarrow \mathbb{R} \cup\{+\infty\}$ be a convex and lower semicontinuous function such that $K_{\phi} \neq \emptyset$. Further, suppose that $j$ satisfies the conditions (8) and (9) or (8) and (10)-(11). Then $S^{\prime} \subset S$. Moreover, if $j$ is regular at $\left(x, u_{0}(x)\right)$ for all $u_{0} \in S^{\prime}$ and $x \in \Omega$, then $S^{\prime}=S$.

Proof. For any $u_{0} \in S^{\prime}$, there exists $u_{0}^{*} \in F\left(u_{0}\right)$ such that

$$
\left\langle u_{0}^{*}, v-u_{0}\right\rangle+\phi(v)-\phi\left(u_{0}\right)+J^{\circ}\left(\widehat{u}_{0} ; \widehat{v}-\widehat{u}_{0}\right) \geq 0, \quad \forall v \in K .
$$

Since $J(\varphi)=\int_{\Omega} j(x, \varphi(x)) d x$, and $j$ satisfies the conditions (8) and (9) or (8) and (10)-(11), by Lemma 4 , we have

$$
\int_{\Omega} j^{\circ}(x, \widehat{u}(x) ; \widehat{v}(x)-\widehat{u}(x)) d x \geq J^{\circ}(\widehat{u} ; \widehat{v}-\widehat{u}),
$$

$$
\forall u, v \in \mathbb{R}^{n}
$$

Then we have

$$
\begin{aligned}
&\left\langle u_{0}^{*}, v-u_{0}\right\rangle+\phi(v)-\phi\left(u_{0}\right) \\
& \quad+\int_{\Omega} j^{\circ}\left(x, \widehat{u}_{0}(x) ; \widehat{v}(x)-\widehat{u}_{0}(x)\right) d x \geq 0, \quad \forall v \in K .
\end{aligned}
$$

That is, $u_{0} \in S$.

If $j$ is regular at $\left(x, u_{0}(x)\right)$, for any $u_{0} \in S^{\prime}$ and $x \in \Omega$, by Lemma 4 , we have

$$
\int_{\Omega} j^{\circ}\left(x, \widehat{u}_{0}(x) ; \widehat{v}(x)-\widehat{u}_{0}(x)\right) d x=J^{\circ}\left(\widehat{u}_{0} ; \widehat{v}-\widehat{u}_{0}\right),
$$

$\forall v \in X$.

Hence, it follows that $S^{\prime}=S$. This completes the proof.

Theorem 10. Let $J: L^{p}\left(\Omega ; \mathbb{R}^{k}\right) \rightarrow \mathbb{R}$ be the function

$$
J(\varphi)=\int_{\Omega} j(x, \varphi(x)) d x .
$$

And let $T: \mathbb{R}^{n} \rightarrow L^{p}\left(\Omega ; \mathbb{R}^{k}\right)$ be a linear compact operator, where $1<p<\infty, k \geq 1$, and $\Omega$ is a bounded open set in $\mathbb{R}^{N}$. Assume that $K$ is a nonempty, closed, and convex subset of $\mathbb{R}^{n}$. Let $\phi: \mathbb{R}^{n} \rightarrow \mathbb{R} \cup\{+\infty\}$ be a convex and lower semicontinuous function such that $K_{\phi} \neq \emptyset$ and let $F: K \rightrightarrows \mathbb{R}^{n}$ be an upper semicontinuous set-valued mapping with compact convex values. Further, suppose that $j$ satisfies the conditions (8) and (9) or (8) and (10)-(11). If the following coercivity condition holds:
(C1) there exists a vector $\widehat{u} \in K_{\phi}$ such that $\phi(\widehat{u})=$ $\inf _{x \in K_{\phi}} \phi(x)$ and the set

$$
\left\{u \in K_{\phi}: \inf _{u^{*} \in\left(F+T^{*} \circ \partial J \circ T\right)(u)}\left\langle u^{*}, v-\widehat{u}\right\rangle+\phi(v)-\phi(\widehat{u})<0\right\}
$$

is bounded (possibly empty), then problem $\left(P^{\prime}\right)$ has at least one solution.

Proof. For the sake of convenience, denote a set-valued mapping $G: K \rightrightarrows \mathbb{R}^{n}$ as follows:

$$
G(u):=\left(F+T^{*} \circ \partial J \circ T\right)(u), \quad \forall u \in K .
$$

Then $G$ is an upper semicontinuous mapping with compact convex values on $K$. In fact, since $j$ satisfies the conditions either (8) and (9) or (8) and (10)-(11), by Lemma $4, J$ is uniformly Lipschitz on $K$, and by item (iii) of Lemma 3, we obtain that $\partial J$ is upper semicontinuous, by the assumption of $T$ being a linear compact operator and $T^{*}$ being its adjoint operator, it follows that $T^{*} \circ \partial J \circ T$ is upper semicontinuous. Since the sum of upper semicontinuous mappings is also upper semicontinuous, by the assumption of $F$ being upper semicontinuous, we have that $G$ is upper semicontinuous. By item (i) of Lemma 3 and the assumptions of $T$ being a linear compact operator and $F$ having compact convex values, we know that $\partial J$ has compact convex values. Thus, $G$ has compact convex values.

Hence, from Theorem 7, we know that there exist $u_{0} \in$ $K_{\phi}$ and $u_{0}^{*} \in G\left(u_{0}\right)$ such that

$$
\left\langle u_{0}^{*}, v-u_{0}\right\rangle+\phi(v)-\phi\left(u_{0}\right) \geq 0, \quad \forall v \in K .
$$

It follows from the definition of $G$ that there exist $u_{1}^{*} \in F\left(u_{0}\right)$ and $u_{2}^{*} \in\left(T^{*} \circ \partial J \circ T\right)\left(u_{0}\right)$ such that $u_{0}^{*}=u_{1}^{*}+u_{2}^{*}$. Then we have that

$$
\left\langle u_{1}^{*}, v-u_{0}\right\rangle+\left\langle u_{2}^{*}, v-u_{0}\right\rangle+\phi(v)-\phi\left(u_{0}\right) \geq 0, \quad \forall v \in K .
$$

By Lemma 3(ii), we have that

$$
\begin{aligned}
J^{\circ}(\widehat{u} ; \widehat{v}-\widehat{u}) & =\max _{\xi \in \partial J(\widehat{u})}\langle\xi, \widehat{v}-\widehat{u}\rangle=\max _{\xi \in \partial J(\widehat{u})}\left\langle T^{*}(\xi), v-u\right\rangle \\
& =\max _{\eta \in T^{*}(\partial J(\hat{u}))}\langle\eta, v-u\rangle .
\end{aligned}
$$

Hence, we have that there exist $u_{0} \in K_{\phi}$ and $u_{1}^{*} \in F\left(u_{0}\right)$ such that

$$
\left\langle u_{1}^{*}, v-u_{0}\right\rangle+\phi(v)-\phi\left(u_{0}\right)+J^{\circ}\left(\widehat{u}_{0} ; \widehat{v}-\widehat{u}_{0}\right), \quad \forall v \in K .
$$

That is, problem $\left(\mathrm{P}^{\prime}\right)$ has at least one solution. This completes the proof.

From Theorem 10 and Proposition 9, we get an existence result of problem $(\mathrm{P})$. 
Theorem 11. Let $J: L^{p}\left(\Omega ; \mathbb{R}^{k}\right) \rightarrow \mathbb{R}$ be the function

$$
J(\varphi)=\int_{\Omega} j(x, \varphi(x)) d x
$$

And let $T: \mathbb{R}^{n} \rightarrow L^{p}\left(\Omega ; \mathbb{R}^{k}\right)$ be a linear compact operator, where $1<p<\infty, k \geq 1$ and $\Omega$ is a bounded open set in $\mathbb{R}^{N}$. Assume that $K$ is a nonempty, closed, and convex subset of $\mathbb{R}^{n}$. Let $\phi: \mathbb{R}^{n} \rightarrow \mathbb{R} \cup\{+\infty\}$ be a convex and lower semicontinuous function such that $K_{\phi} \neq \emptyset$ and $F: K \rightrightarrows \mathbb{R}^{n}$ be an upper semicontinuous set-valued mapping with compact convex values. Further, suppose that $j$ satisfies the conditions (8) and (9) or (8) and (10)-(11). If coercivity condition (C1) holds, then problem $(P)$ has at least one solution.

Corollary 12. Assume that $K$ is a nonempty, bounded, closed, and convex subset of $\mathbb{R}^{n}$. Let $\phi: \mathbb{R}^{n} \rightarrow \mathbb{R}$ be a convex and lower semicontinuous function and $F: K \rightrightarrows \mathbb{R}^{n}$ be an upper semicontinuous set-valued mapping with compact convex values. Further, suppose that $j$ satisfies the conditions (8) and (9) or (8) and (10)-(11). Then problem (P) has at least one solution.

Proof. Let $J: L^{p}\left(\Omega ; \mathbb{R}^{k}\right) \rightarrow \mathbb{R}$ be the function

$$
J(\varphi)=\int_{\Omega} j(x, \varphi(x)) d x .
$$

By the lower semicontinuity of $\phi$, we know that there exists a vector $\widehat{u} \in K_{\phi}$ such that $\phi(\widehat{u})=\inf _{x \in K_{\phi}} \phi(x)$. It follows from the boundedness of $K$ that

$$
\left\{u \in K_{\phi}: \inf _{u^{*} \in\left(F+T^{*} \circ \partial J \circ T\right)(u)}\left\langle u^{*}, v-\widehat{u}\right\rangle+\phi(v)-\phi(\widehat{u})<0\right\}
$$

is bounded (possibly empty). Thus, from Theorem 15, we know that problem $(\mathrm{P})$ has at least one solution. This completes the proof.

Remark 13. Corollary 12 generalizes and improves some recent results in the following aspects.

(i) If $\phi=0$, then Corollary 12 reduces to Theorem 3.2 of Zhang and $\mathrm{He}$ [5].

(ii) Compared with Theorem 4.1 of Tang and Huang [2], the main difference lies in the assumptions on $F$. Theorem 4.1 of Tang and Huang [2] asked $F$ to be a lower hemicontinuous and $\phi$-quasimonotone mapping.

Proposition 14 (Proposition 4.1 of [2]). Consider the following coercivity conditions.

(C2) There exists a nonempty subset $V_{0}$ contained in a weakly compact subset $V_{1}$ of $K_{\phi}$ such that the set

$$
\begin{aligned}
D=\left\{u \in K_{\phi}: \inf _{v^{*} \in F(v)}\left\langle v^{*}, v-u\right\rangle+\phi(v)-\phi(u)\right. \\
\left.+J^{0}(\widehat{u} ; \widehat{v}-\widehat{u}) \geq 0, v \in V_{0}\right\}
\end{aligned}
$$

(C3) There exist $n_{0}>0$ and $n_{0} \in \mathbb{N}$ such that for every $u \in$ $K_{\phi} \backslash B_{n_{0}}$, there exists some $v \in K_{\phi}$ with $\|v\|<\|u\|$ such that

$$
\sup _{u^{*} \in F(u)}\left\langle u^{*}, v-u\right\rangle+\phi(v)-\phi(u)+J^{0}(\widehat{u} ; \widehat{v}-\widehat{u}) \leq 0 .
$$

(C4) There exist $n_{0}>0$ and $n_{0} \in \mathbb{N}$ such that for every $u \in$ $K_{\phi} \backslash B_{n_{0}}$, there exists some $v \in K_{\phi}$ with $\|v\|<\|u\|$ such that

$$
\begin{aligned}
\sup _{u^{*} \in F(u)}\left\langle u^{*}, v-u\right\rangle+\phi(v)-\phi(u) \\
\quad+\int_{\Omega} j^{0}(x, \widehat{u}(x) ; \widehat{v}(x)-\widehat{u}(x)) d x<0 .
\end{aligned}
$$

Then we have

(i) $(\mathrm{C} 2) \Rightarrow(\mathrm{C} 3)$, if $F$ is stably $\phi$-quasimonotone with respect to the set $U(J, T)$;

(ii) $(C 4) \Rightarrow(C 3)$, if $J(\varphi)=\int_{\Omega} j(x, \varphi(x)) d x, j$ satisfies the conditions (8) and (9) or (8) and (10)-(11).

Theorem 15. Let $J: L^{p}\left(\Omega ; \mathbb{R}^{k}\right) \rightarrow \mathbb{R}$ be the function

$$
J(\varphi)=\int_{\Omega} j(x, \varphi(x)) d x .
$$

And let $T: \mathbb{R}^{n} \rightarrow L^{p}\left(\Omega ; \mathbb{R}^{k}\right)$ be a linear compact operator, where $1<p<\infty, k \geq 1$, and $\Omega$ is a bounded open set in $\mathbb{R}^{N}$. Assume that $K$ is a nonempty, closed, and convex subset of $\mathbb{R}^{n}$ and $\phi: \mathbb{R}^{n} \rightarrow \mathbb{R} \cup\{+\infty\}$ is a convex and lower semicontinuous function such that $K_{\phi} \neq \emptyset$. Let $F: K \rightrightarrows \mathbb{R}^{n}$ be an upper semicontinuous set-valued mapping with compact convex values. If the condition $(C 3)$ holds, then the problem $(P)$ admits at least one solution.

Proof. Take $m>n_{0}$ and $B_{m}=\{u: u \in K \cap \operatorname{dom} \phi$ and $\|u\| \leq$ $m$ \}. From Proposition 9 and Corollary 12, we conclude that there exist $u_{m} \in B_{m}$ and $u_{m}^{*} \in F\left(u_{m}\right)$ such that

$$
\begin{array}{r}
\left\langle u_{m}^{*}, v-u_{m}\right\rangle+\phi(v)-\phi\left(u_{m}\right)+J^{0}\left(\widehat{u}_{m} ; \widehat{v}-\widehat{u}_{m}\right) \geq 0, \\
\forall v \in B_{m} .
\end{array}
$$

(i) If $\left\|u_{m}\right\|=m$, then $\left\|u_{m}\right\|>n_{0}$. Since the condition (C3) holds, there is some $v_{0} \in K_{\phi}$ with $\left\|v_{0}\right\|<\left\|u_{m}\right\|$ such that

$$
\left\langle u_{m}^{*}, v_{0}-u_{m}\right\rangle+\phi\left(v_{0}\right)-\phi\left(u_{m}\right)+J^{0}\left(\widehat{u}_{m} ; \widehat{v}_{0}-\widehat{u}_{m}\right) \leq 0 .
$$

Let $v \in K_{\phi}$ be arbitrarily fixed. Since $\left\|v_{0}\right\|<\left\|u_{m}\right\|=m$, there is $t \in(0,1)$ such that $v_{t}:=v_{0}+t\left(v-v_{0}\right) \in B_{m}$. Note that $T$ is a linear mapping and $\phi$ is convex. It follows from (41), (42) and Lemma 2(i) that

$$
\begin{aligned}
0 \leq & \left\langle u_{m}^{*}, v_{t}-u_{m}\right\rangle+\phi\left(v_{t}\right)-\phi\left(u_{m}\right)+J^{0}\left(\widehat{u}_{m} ; \widehat{v}_{t}-\widehat{u}_{m}\right) \\
= & \left\langle u_{m}^{*},(1-t) v_{0}+t v-u_{m}\right\rangle+\phi\left((1-t) v_{0}+t v\right)-\phi\left(u_{m}\right) \\
& +J^{0}\left(\widehat{u}_{m} ;(1-t) \widehat{v}_{0}+t \widehat{v}-\widehat{u}_{m}\right)
\end{aligned}
$$




$$
\begin{array}{r}
\leq(1-t)\left[\left\langle u_{m}^{*}, v_{0}-u_{m}\right\rangle+\phi\left(v_{0}\right)\right. \\
\left.-\phi\left(u_{m}\right)+J^{0}\left(\widehat{u}_{m} ; \widehat{v}_{0}-\widehat{u}_{m}\right)\right] \\
+t\left[\left\langle u_{m}^{*}, v-u_{m}\right\rangle+\phi(v)-\phi\left(u_{m}\right)+J^{0}\left(\widehat{u}_{m} ; \widehat{v}-\widehat{u}_{m}\right)\right] \\
\leq t\left[\left\langle u_{m}^{*}, v-u_{m}\right\rangle+\phi(v)-\phi\left(u_{m}\right)+J^{0}\left(\widehat{u}_{m} ; \widehat{v}-\widehat{u}_{m}\right)\right], \\
\forall v \in K_{\phi} .
\end{array}
$$

Therefore, this together with $t \in(0,1)$ implies that

$$
\begin{array}{r}
\left\langle u_{m}^{*}, v-u_{m}\right\rangle+\phi(v)-\phi\left(u_{m}\right)+J^{0}\left(\widehat{u}_{m} ; \widehat{v}-\widehat{u}_{m}\right) \geq 0, \\
\forall v \in K_{\phi} .
\end{array}
$$

(ii) If $\left\|u_{m}\right\|<m$, then for any $v \in K_{\phi}$, there is some $t \in$ $(0,1)$ such that $v_{t}:=u_{m}+t\left(v-v_{m}\right) \in B_{m}$. Note that $T$ is a linear mapping and $\phi$ is a convex function. It follows from (41) and item (i) of Lemma 2 that

$$
\begin{array}{r}
0 \leq\left\langle u_{m}^{*}, v_{t}-u_{m}\right\rangle+\phi\left(v_{t}\right)-\phi\left(u_{m}\right)+J^{0}\left(\widehat{u}_{m} ; \widehat{v}_{t}-\widehat{u}_{m}\right) \\
\leq t\left[\left\langle u_{m}^{*}, v-u_{m}\right\rangle+\phi(v)-\phi\left(u_{m}\right)+J^{0}\left(\widehat{u}_{m} ; \widehat{v}-\widehat{u}_{m}\right)\right], \\
\forall v \in K_{\phi} .
\end{array}
$$

Therefore, this together with $t \in(0,1)$ implies that (44) also holds.

Since $J(\varphi)=\int_{\Omega} j(x, \varphi(x)) d x$ and $j$ satisfies the conditions (8) and (9) or (8) and (10)-(11), by Lemma 4, we have

$$
\int_{\Omega} j^{0}(x, \widehat{u}(x) ; \widehat{v}(x)-\widehat{u}(x)) d x \geq J^{0}(\widehat{u} ; \widehat{v}-\widehat{u}), \quad \forall u, v \in X
$$

and so

$$
\begin{aligned}
& \left\langle u_{m}^{*}, v-u_{m}\right\rangle+\phi(v)-\phi\left(u_{m}\right) \\
& \quad+\int_{\Omega} j^{0}\left(x, \widehat{u}_{m}(x) ; \widehat{v}(x)-\widehat{u}_{m}(x)\right) d x \\
& \geq\left\langle u_{m}^{*}, v-u_{m}\right\rangle+\phi(v)-\phi\left(u_{m}\right) \\
& \quad+J^{0}\left(\widehat{u}_{m} ; \widehat{v}-\widehat{u}_{m}\right) \geq 0, \quad \forall v \in K_{\phi} .
\end{aligned}
$$

If $v^{\prime} \in K \backslash \operatorname{dom} \phi$, then $\phi\left(v^{\prime}\right)=+\infty$ and thus the inequality in (47) holds automatically. This together with (47) shows that $u_{m} \in K_{\phi}$ is a solution of problem (P).

Remark 16. Theorem 15 generalizes and improves some recent results in the following aspects.

(i) If $\phi=I_{K}$, then Theorem 15 reduces to Theorem 3.3 of [5]. (ii) Compared with Theorem 4.2 of Tang and Huang [2], the main difference lies in the assumptions on $F$. Theorem 4.2 of Tang and Huang [2] asked $F$ to be a lower hemicontinuous and $\phi$-quasimonotone mapping.

If the constraint set $K$ is bounded, then the solution set of the problem $(\mathrm{P})$ is obviously bounded. In the case when the constraint set $K$ is unbounded, the solution set of the problem (P) may be unbounded. In the sequel, we provide a sufficient condition to the boundedness of the solution set of the problem (P), when $K$ is unbounded. The following theorem also generalizes Theorem 4.1 of [5].

Theorem 17. Let $J: L^{p}\left(\Omega ; \mathbb{R}^{k}\right) \rightarrow \mathbb{R}$ be the function

$$
J(\varphi)=\int_{\Omega} j(x, \varphi(x)) d x .
$$

and $T: \mathbb{R}^{n} \rightarrow L^{p}\left(\Omega ; \mathbb{R}^{k}\right)$ be a linear compact operator, where $1<p<\infty, k \geq 1$, and $\Omega$ is a bounded open set in $\mathbb{R}^{N}$. Assume that $K$ is a nonempty, closed, and convex subset of $\mathbb{R}^{n}$ and $\phi$ : $\mathbb{R}^{n} \rightarrow \mathbb{R} \cup\{+\infty\}$ is a convex and lower semicontinuous function such that $K_{\phi} \neq \emptyset$. Let $F: K \rightrightarrows \mathbb{R}^{n}$ be an upper semicontinuous set-valued mapping with compact convex values. Further, we suppose $j$ satisfies the conditions (8) and (9) or (8) and (10)(11). If condition (C4) holds, then the solution set of problem (P) is nonempty and bounded.

Proof. From Proposition 14, we have $(\mathrm{C} 4) \Rightarrow(\mathrm{C} 3)$. By Theorem 15, we know that the solution set of the problem (P) is nonempty. If the solution set is unbounded, then there exist $u_{0} \in K_{\phi}$ and $u_{0}^{*} \in F\left(u_{0}\right)$ such that $\left\|u_{0}\right\|>n_{0}$ and

$$
\begin{aligned}
&\left\langle u_{0}^{*}, v-u_{0}\right\rangle+\phi(v)-\phi\left(u_{0}\right) \\
& \quad+\int_{\Omega} j^{0}\left(x, \widehat{u}_{0}(x) ; \widehat{v}(x)-\widehat{u}_{0}(x)\right) d x \geq 0, \quad \forall v \in K .
\end{aligned}
$$

Since $\left\|u_{0}\right\|>n_{0}$, by the condition (C4), there exists $v_{0} \in K_{\phi}$ with $\left\|v_{0}\right\|<\left\|u_{0}\right\|$ such that

$$
\begin{aligned}
\sup _{u^{*} \in F\left(u_{0}\right)}\left\langle u^{*}, v_{0}-u_{0}\right\rangle+\phi\left(v_{0}\right)-\phi\left(u_{0}\right) \\
+\int_{\Omega} j^{0}\left(x, \widehat{u}_{0}(x) ; \widehat{v}_{0}(x)-\widehat{u}_{0}(x)\right) d x<0,
\end{aligned}
$$

which contradicts (49). Hence, the solution set is bounded.

\section{Conflict of Interests}

The authors declare that they have no conflict of interests regarding the publication of this paper.

\section{Acknowledgments}

Guo-ji Tang was partially supported by Guangxi Natural Science Foundation (2013GXNSFBA019015), Scientific 
Research Foundation of Guangxi University for Nationalities (2012QD015), and Open fund of Guangxi key laboratory of hybrid computation and IC design analysis (HCIC201308). Hong-ling Zhang was partially supported by the Key Program of the Fundamental Research Funds for the Central Universities (A0920502051202-111).

\section{References}

[1] N. Costea and C. Lupu, "On a class of variational-hemivariational inequalities involving set valued mappings," Advances in Pure and Applied Mathematics, vol. 1, no. 2, pp. 233-246, 2010.

[2] G.-J. Tang and N.-J. Huang, "Existence theorems of the variational-hemivariational inequalities," Journal of Global Optimization, vol. 56, no. 2, pp. 605-622, 2013.

[3] D. Motreanu and V. Rădulescu, "Existence results for inequality problems with lack of convexity," Numerical Functional Analysis and Optimization, vol. 21, no. 7-8, pp. 869-884, 2000.

[4] Y. Zhang and Y. He, "On stably quasimonotone hemivariational inequalities," Nonlinear Analysis: Theory, Methods \& Applications, vol. 74, no. 10, pp. 3324-3332, 2011.

[5] Y. L. Zhang and Y. R. He, "The hemivariational inequalities for an upper semicontinuous set-valued mapping," Journal of Optimization Theory and Applications, vol. 156, no. 3, pp. 716725, 2013.

[6] P. D. Panagiotopoulos, M. Fundo, and V. Rădulescu, "Existence theorems of Hartman-Stampacchia type for hemivariational inequalities and applications," Journal of Global Optimization, vol. 15, no. 1, pp. 41-54, 1999.

[7] N. Costea and V. Rădulescu, "Hartman-stampacchia results for stably pseudomonotone operators and non-linear hemivariational inequalities," Applicable Analysis, vol. 89, no. 2, pp. 175$188,2010$.

[8] G.-J. Tang and N.-J. Huang, "Projected subgradient method for non-Lipschitz set-valued mixed variational inequalities," Applied Mathematics and Mechanics, vol. 32, no. 10, pp. 13451356, 2011.

[9] G.-J. Tang and N.-J. Huang, "Strong convergence of an inexact projected subgradient method for mixed variational inequalities," Optimization, vol. 63, no. 4, pp. 601-615, 2014.

[10] Z. B. Wang and N. J. Huang, "Degree theory for a generalized set-valued variational inequality with an application in Banach spaces," Journal of Global Optimization, vol. 49, no. 2, pp. 343357, 2011.

[11] F.-Q. Xia and N.-J. Huang, "An inexact hybrid projection-proximal point algorithm for solving generalized mixed variational inequalities," Computers and Mathematics with Applications, vol. 62 , no. 12, pp. 4596-4604, 2011.

[12] R.-Y. Zhong and N.-J. Huang, "Stability analysis for minty mixed variational inequality in reflexive Banach spaces," Journal of Optimization Theory and Applications, vol. 147, no. 3, pp. 454472, 2010.

[13] P. D. Panagiotopoulos, "Nonconvex energy functions. Hemivariational inequalities and substationarity principles," Acta Mechanica, vol. 48, no. 3-4, pp. 111-130, 1983.

[14] P. D. Panagiotopoulos, Inequality Problems in Mechanics and Applications: Convex and Nonconvex Energy Functions, Birkhäuser, Basel, Switzerland, 1985.

[15] P. D. Panagiotopoulos, "Coercive and semicoercive hemivariational inequalities," Nonlinear Analysis, vol. 16, no. 3, pp. 209231, 1991.
[16] P. D. Panagiotopoulos, Hemivariational Inequalities: Applications in Mechnics and Engineering, Springer, Berlin, Germany, 1993.

[17] F. H. Clarke, Optimization and Nonsmooth Analysis, John Wiley \& Sons, New York, NY, USA, 1983.

[18] Z. Naniewicz and P. D. Panagiotopoulos, Mathematical Theory of Hemivariational Inequalities and Applications, Marcel Dekker, New York, NY, USA, 1995.

[19] S. Carl, "Existence of extremal solutions of boundary hemivariational inequalities," Journal of Differential Equations, vol. 171, no. 2, pp. 370-396, 2001.

[20] S. Carl, V. K. Le, and D. Motreanu, "Existence and comparison principles for general quasilinear variational-hemivariational inequalities," Journal of Mathematical Analysis and Applications, vol. 302, no. 1, pp. 65-83, 2005.

[21] S. Carl, V. K. Le, and D. Motreanu, Nonsmooth Variational Problems and Their Inequalities: Comparison Principles and Applications, Springer, New York, NY, USA, 2007.

[22] Y.-B. Xiao and N.-J. Huang, "Sub-super-solution method for a class of higher order evolution hemivariational inequalities," Nonlinear Analysis: Theory, Methods \& Applications, vol. 71, no. 1-2, pp. 558-570, 2009.

[23] S. Migórski and A. Ochal, "Boundary hemivariational inequality of parabolic type," Nonlinear Analysis: Theory, Methods \& Applications, vol. 57, no. 4, pp. 579-596, 2004.

[24] J. Y. Park and T. G. Ha, "Existence of antiperiodic solutions for hemivariational inequalities," Nonlinear Analysis: Theory, Methods \& Applications, vol. 68, no. 4, pp. 747-767, 2008.

[25] J. Y. Park and T. G. Ha, "Existence of anti-periodic solutions for quasilinear parabolic hemivariational inequalities," Nonlinear Analysis: Theory, Methods \& Applications, vol. 71, no. 7-8, pp. 3203-3217, 2009.

[26] D. Goeleven, D. Motreanu, and P. D. Panagiotopoulos, "Eigenvalue problems for variational-hemivariational inequalities at resonance," Nonlinear Analysis: Theory, Methods \& Applications, vol. 33, no. 2, pp. 161-180, 1998.

[27] Z. Liu, "Existence results for quasilinear parabolic hemivariational inequalities," Journal of Differential Equations, vol. 244, no. 6, pp. 1395-1409, 2008.

[28] N. Costea, "Existence and uniqueness results for a class of quasihemivariational inequalities," Journal of Mathematical Analysis and Applications, vol. 373, no. 1, pp. 305-315, 2011.

[29] Z. Peng and Z. Liu, "Evolution hemivariational inequality problems with doubly nonlinear operators," Journal of Global Optimization, vol. 51, no. 3, pp. 413-427, 2011.

[30] Y.-B. Xiao and N.-J. Huang, "Well-posedness for a class of variational-hemivariational inequalities with perturbations," Journal of Optimization Theory and Applications, vol. 151, no. 1, pp. 33-51, 2011.

[31] M. Bianchi and R. Pini, "Coercivity conditions for equilibrium problems," Journal of Optimization Theory and Applications, vol. 124, no. 1, pp. 79-92, 2005.

[32] A. Daniilidis and N. Hadjisavvas, "Coercivity conditions and variational inequalities," Mathematical Programming B, vol. 86, no. 2, pp. 433-438, 1999.

[33] F. Facchinei and J.-S. Pang, Finite-Dimensional Variational Inequalities and Complementarity Problems, vol. I, Springer, New York, NY, USA, 2003.

[34] I. V. Konnov and D. A. Dyabilkin, "Nonmonotone equilibrium problems: coercivity conditions and weak regularization," Journal of Global Optimization, vol. 49, no. 4, pp. 575-587, 2011. 
[35] K. Deimling, Nonlinear Functional Analysis, Springer, Berlin, Germany, 1985.

[36] F. Qiao and Y. He, "Strict feasibility of pseudomonotone setvalued variational inequalities," Optimization, vol. 60, no. 3, pp. 303-310, 2011. 


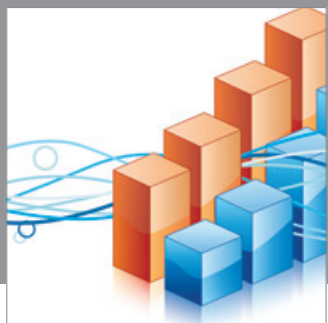

Advances in

Operations Research

mansans

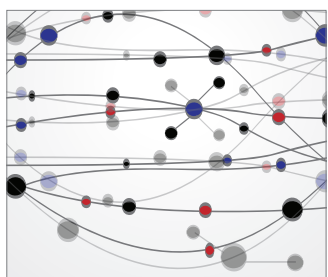

The Scientific World Journal
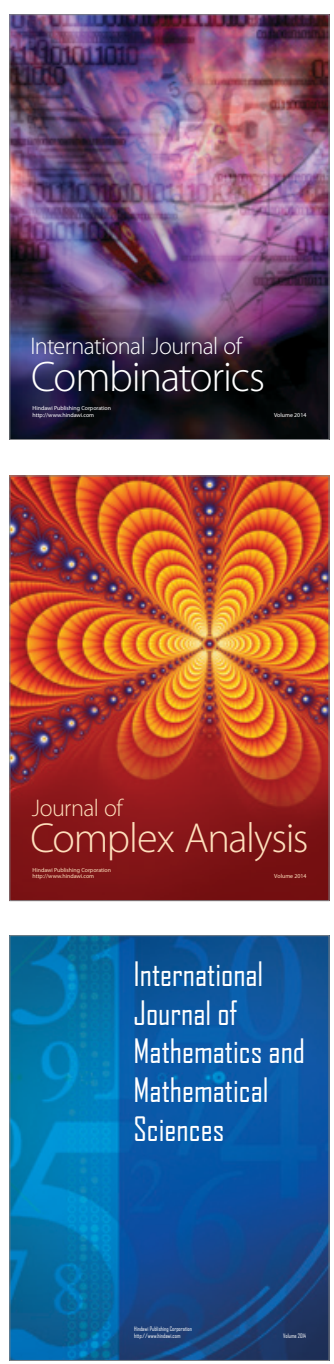
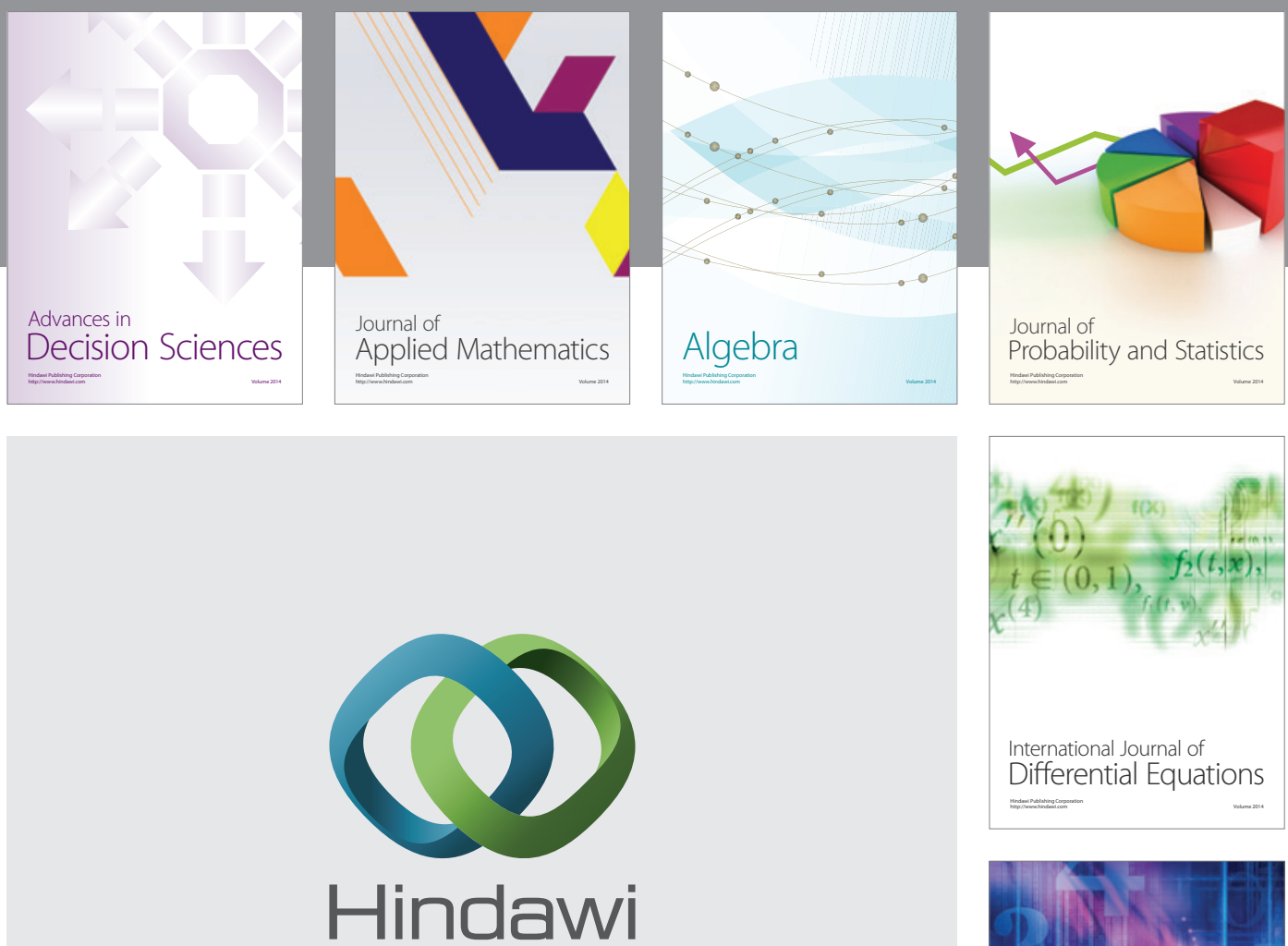

Submit your manuscripts at http://www.hindawi.com
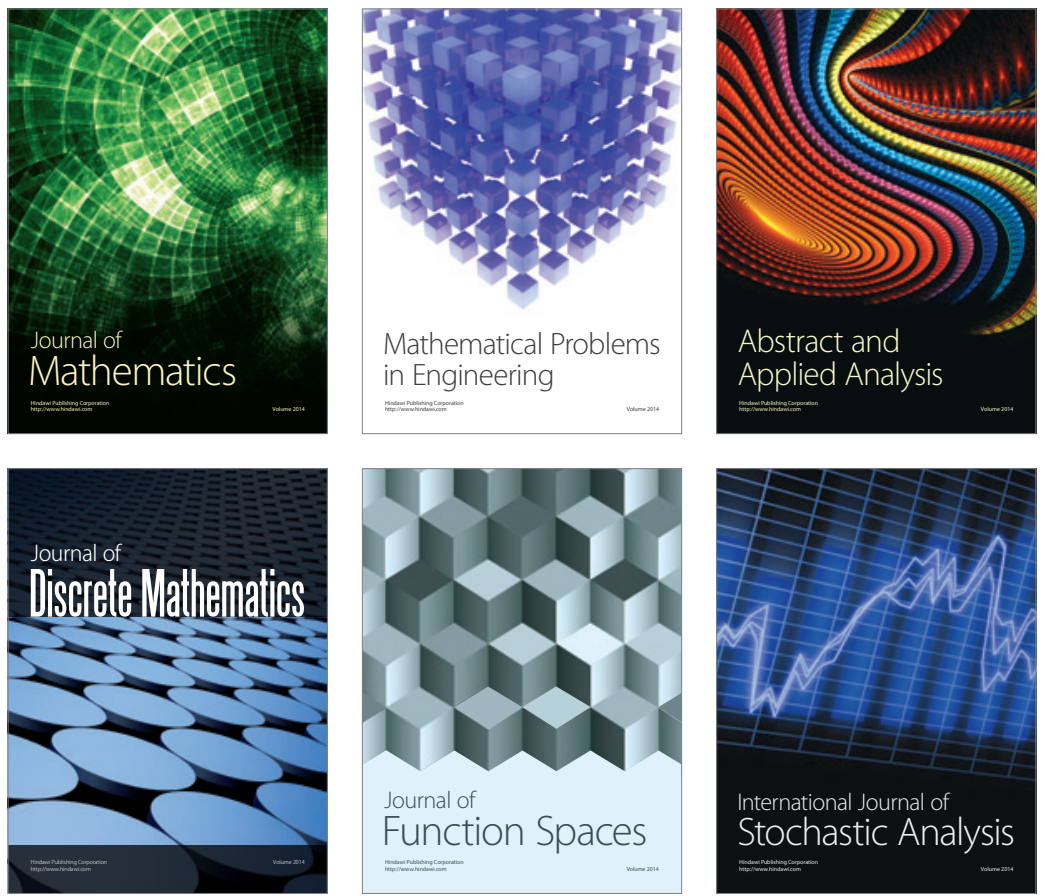

Journal of

Function Spaces

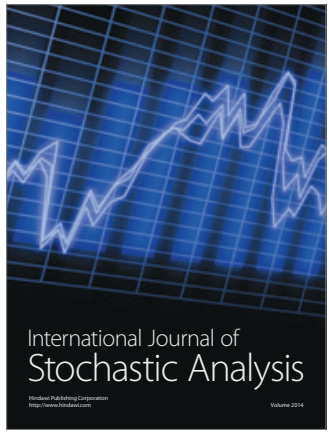

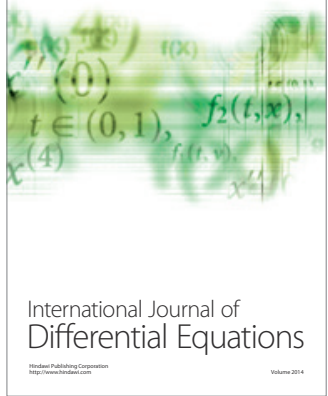
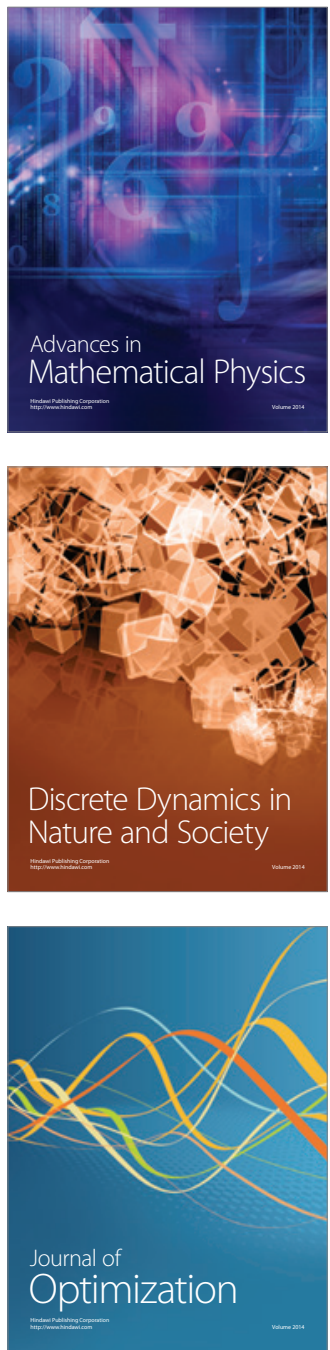